mimic physiological TME to dissect the combinational role of Nivolumab and Blinatumomab (figure 2).

Results The result suggest that combinatory therapy is highly depend on the dosage of Blinatumomab and also $\mathrm{T}$ cell number in the TME, which might give an instruction for ongoing clinical trial design. Finally, we have employed humanized mouse models bearing Raji or Daudi tumor cells to further validate this combination treatment in vivo. Both In-vivo and In-vitro data support that Blinatumomab is dominant in activing $\mathrm{T}$ cell and Nivolumab can only exhibit synergistic effect under suboptimal dosage of Blinatumomab.

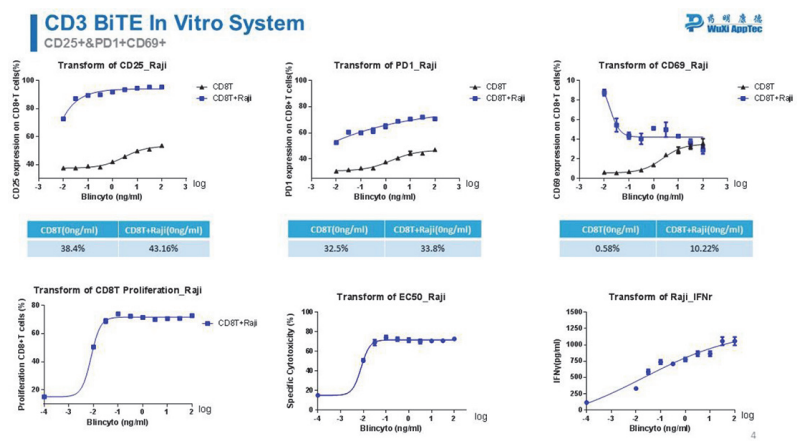

Abstract 781 Figure 1 Establishment of In vitro co-culture system for CD3 BiTE establish in vitro human PBMC based system to validate CD3 BiTE function
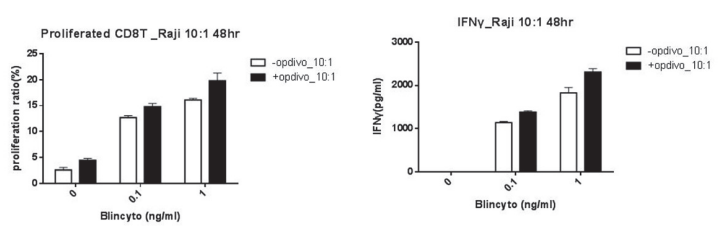

Abstract 781 Figure 2 Opdivo and CD3 BiTE Combination Opdivo could further promote T cell activation under the treatment of CD3 BiTE

Conclusions Successfully establish in vitro system to evaluate the function of CD3 BiTE and also take advantage of MLR/ tumor co-culture system to demonstrate PD1 antibody could further promote $\mathrm{T}$ cell activation under appropriate dosage of CD3 BiTE.

http://dx.doi.org/10.1136/jitc-2020-SITC2020.0781

\section{ALDH1A INHIBITION AS ADJUVANT TO OVARIAN CANCER IMMUNOTHERAPY}

${ }^{1}$ Bingsi Gao*, ${ }^{2}$ Mainpal Rana, ${ }^{2}$ Dongli Yang, ${ }^{2}$ Edward Grimley, ${ }^{2}$ Stacy McGonigal, ${ }^{3}$ Ronald Buckanovich, ${ }^{2}$ Anda Vlad. 'University of Pittsburgh, Magee Womens Research Institute, Third Xiangya Hospital of CSU, Pittsburgh, PA, USA; ${ }^{2}$ University of Pittsburgh, Magee Womens Research Institute, Pittsbutgh, PA, USA; ${ }^{3}$ University of Pittsburgh, Magee Womens Research Institute, UPMC Hillman Cancer Center, Pittsbutgh, PA, USA

Background Despite some recent advances, new therapeutic approaches for ovarian cancer (OvCa), the 5th leading cause of cancer deaths in women, are clearly needed. Aldehyde dehydrogenase-1A (ALDH1A) enzymes represent a novel therapeutic target for OvCa. ALDH1A is upregulated in OvCa initiating cells and mediate the biosynthesis of retinoic acid (RA) to regulate numerous cellular processes, including proliferation, metastasis, and chemotherapy resistance. We recently identified novel pan-ALDH1A family inhibitors (ALDHi) that induce necroptosis in OvCa stem-like cells and synergize with chemotherapy, leading to tumor eradication in vivo. Here, we hypothesize that, in addition to controlling tumor progression, ALDHi trigger immunogenic cell death (ICD) via necroptosis and can potentiate anti-tumor immunity.

Methods We performed RNA-Seq on four human OvCa cell lines (A2780, CAOV-3, OVCAR-5, OVSAHO) treated for 8 hours with two different ALDHi. To measure the impact on $\mathrm{T}$ cell immunity we performed flow cytometry to measure cell proliferation assays and CD4 naïve differentiation into Th1/ Th17/Treg subsets. Molecular targets in the RA pathway were confirmed by western blot.

Results ALDHi triggered significant changes in (i) ER stress unfolded protein response and regulators of the ER stress response, such as ATF4 and EIF2aK3 (PERK), (ii) inflammatory pathways, (iii) cell death, survival, and (iv) gene transcription-RAR signaling. Treatment of cancer cell lines with ALDHi induced expression of Phospho-eIF2 $\alpha$, a marker for the ICD, along with increased expression of ATF3 and ATF4, and calreticulin, suggesting cancer cells undergoing ICD.

Using polyclonal stimulation of murine splenocytes and human PBMC, we observed that ALDHi promote $\mathrm{T}$ cell proliferation, especially of CD8 T cells. Furthermore, exposure of naïve CD4 cells to Th1 and Treg differentiation conditions leads to increased production of INF $\square$ and reduced number of Foxp3 + iTregs, respectively. Further, in a co-culture of iTreg and stimulated splenocyte, ALDHi treatment diminishes the iTreg's capacity to induce immune suppression. Ex vivo treatment of ovarian cancer ascites cells with various ALDHi leads to significant decrease of CD14+ cells, an effect associated with downregulation of NR4A1 (NUR77), a nuclear receptor that interacts RAR/RXR, downstream of RA signaling.

Conclusions ADLHi induce immunogenic cell death in cancer cells. Immune cells respond to ALDHi in a cell specific manner. ALDHi support CD8 T cell proliferation and CD4 Th1 induction, while inhibiting iTregs. Exposure to ALDHi leads to downregulation of NR4A1 and reduction in suppressive macrophage numbers. Our results support the use of ALDHi as immune modulators in ovarian cancer and adjuvants to immunotherapy.

http://dx.doi.org/10.1136/jitc-2020-SITC2020.0782

\section{ESTROGEN-DEPRIVATION PROMOTES TH1 POLARIZATION OF TUMOR-ASSOCIATED T CELLS IN A MOUSE MODEL OF HIGH GRADE SEROUS OVARIAN CANCER}

Daniel Falcon*, Marina Miller, Chelsea Goff, Ichiko Kinjyo, Sarah Adams. University of New Mexico, Albuquerque, NM, USA

Background Immunotherapy has achieved long-term survival in patients with melanoma and other tumors, introducing a new paradigm in cancer treatment. Differential outcomes among men and women receiving immune checkpoint inhibitors implicate sex steroids as modulators of treatment response. Estrogen signaling has a profound impact on $\mathrm{T}$ cell function 